Ann. Génét. Sêl. anim., 1972, 4 (2), 25โ-258.

\title{
NUTRITION OF THE DWARF LAYER ( $\left.{ }^{1}\right)$
}

\author{
J. D. SUMMERS \\ Department of Poultry Science, \\ University of Guelph, \\ Guelph, Ontario (Canada)
}

\section{SUMMARY}

Feeding levels of dietary protein varying from I6 to 23 p. 100 and metabolizable energy levels varying from 2.87 to $3.31 \mathrm{kcal}$ per gram failed to significantly influence egg production or egg size of dwarf layers. An increase in weight of dwarf birds at 6 weeks of age was obtained by feeding thyroprotein, thus suggesting that the dwarf layer may be a hypothyrotic bird.

Body temperatures were $1 / 2^{\circ} \mathrm{C}$ lower for dwarf as compared to normal birds, however, feeding thyroprotein brought the body temperature of the dwarf up to that of the normals.

The dwarf bird contained approximately $5 \mathrm{p}$. Ioo more carcass fat on a dry weight basis than did normal birds. Feeding thyroprotein resulted in a decrease in carcass fat for the dwarf bird while thiouracil feeding increased carcass fat. The very opposite effect was noted for carcass fat of the normal birds.

There was no significant difference in packed cell volume, hemoglobin, red blood cell count, mean corpuscular hemoglobin, total cholesterol, serum protein and serum albumin of dwarf and normal birds. Of the plasma amino acids methionine was significantly lower for the dwarf while all other essentials were similar for the dwarf and normal birds.

Although there has been a lot of discussion about the possibility of using dwarf layers in the commercial egg industry, results to-date with regard to egg production and egg size have not been good enough to make the dwarf a serious contender as a replacement for our present day commercial layer.

In table $I$, is shown the average weight and average weekly feed consumption of egg production type dwarfs reared to 8 weeks of age on a normal 20 p. roo protein starter ration. Body weight and feed consumption are approximately $\mathrm{I} / 3$ lower than that for most of to-day's modern layers. The pullets were randomized into floor and cage pens at 8 weeks of age and fed ad libitum high and low energy growing diets ( $2546 \mathrm{vs}$.

(1) This article has been presented at the Symposium on Dwarf Gene in Poultry, Tours, March 4-5th I97r. and abstracted in World Poultry Sci. J., I97. 
$2776 \mathrm{kcal} \mathrm{ME} / \mathrm{kg}$ ) containing I4 p. Ioo protein to 20 weeks of age. Birds reared in cages were slightly heavier than those reared on the floor (table 2). Weight gain was not affected by the energy level of the ration, however, less food was consumed on the lower energy diet.

At twenty weeks of age the pullets were placed in 8 inch laying cages (one bird per cage) and placed on diets containing I5, I7 and I9 p. Ioo protein. After 3, 28 day periods, when it was evident that there was no difference in response to the diets the levels of protein were increased $4 \mathrm{p}$. IOO to I9, 2I and $23 \mathrm{p}$. IOo. At the end of 5,28 day periods, the level of energy was increased in the diets, since it was felt that perhaps energy rather than protein may have been a limiting factor in these diets. The two extremes in diets are shown in table 3 .

Since there was no significant difference in performance between any of the treatments, the results have been averaged to show the overall performance of the birds from 23 to 53 weeks of age (table 4 ). Feed consumption was very low, however, for the rate of production and egg size obtained, efficiency of protein utilization was extremely good.

Several small tests were conducted using higher additions of DL-methionine and also higher levels of vitamins and trace minerals. All these treatments were without effect in enhancing performance.

In order to try and find some answers as to why the dwarf bird did not respond to increased levels of nutrients work was initiated to try and determine whether the dwarf was a hypothyrotic bird. Normal and dwarf chicks were fed a regular chick starter ration containing protamone $\left({ }^{1}\right)$ or thiouracil. Weight gain and feed consumption values to 6 weeks of age are shown in table 5. A marked improvement in weight gain was observed for the dwarf bird when fed protamone while little or no difference was noted for the normal birds. Thiouracil resulted in a marked depression in performance for both dwarf and normal birds. A similar pattern was noted for shank length (table 6).

Body temperature was recorded for dwarf and normal birds at 8 weeks and 7 months of age. It can be noted in table 7 , that the dwarf birds had a lower body temperature than the normal. Feeding thyroprotein raised the body temperature of the dwarf up to that of the normal bird.

In table 8 is shown carcass composition of normal and dwarf birds at 6 weeks of age. The dwarfs had significantly more body fat than the normals. Thyroprotein feeding reduced the fat content of the dwarf bird but increased body fat of the normal. The opposite effect was noted with the feeding of thiouracil. These results suggest a marked difference in metabolism between the dwarf and normal bird.

It was decided to grow dwarf pullets to 20 weeks of age and attempt to enhance their feed consumption and hence egg production and egg size by the feeding of thyroprotein. Various levels of thyroprotein were fed with little or no success. However, in the latest test (table 9) there appeared to be some indication that thyroprotein supplementation may be of benefit to the dwarf bird.

In an additional experiment with dwarf layers, housed and fed individually and again testing various levels of protein and energy, no response to dietary treatment was noted. However out 4 in table ro is shown the number of hens laying at different

(1) Trade name of thyroprotein contains p. Ioo thyroxine activity. 
rates of production for the above test. Even though there were large differences in composition of the diets employed there was no consistant effect on performance. Number of birds in various clutch sizes was summarized for the dietary treatments (table II). Again there is no difference between the treatments, but the large number of clutch sizes of 3 days or less, indicates the main problem with the dwarf bird. Clutch size must be improved before any major improvement in production can be obtained.

A further study was undertaken to compare various blood constituents of the dwarf with those of normal White Leghorn hens. There was no significant difference in packed cell volume, hemoglobin, red blood cell count, or mean corpuscular hemoglobin concentration (table I2). No difference in total cholesterol, serum protein or serum albumen was also noted for the dwarf and normal birds (table r3).

Plasma amino acids were determined for dwarf and normal chicks 8 hours after feeding. Most amino acids were similar, however, there was a marked decrease in the level of methionine for the dwarf as compared to the normal bird. Further work is underway to verify this finding and expand this area of investigation.

Rę̧u pour publication en septembre 1971.

\section{RÉSUMÉ}

\section{NUTRITION DE LA POULE NAINE}

Quoiqu'on ait beaucoup discuté autour de la possibilité d'utiliser des pondeuses naines pour la production commerciale d'œufs, les résultats de ponte et de taille des œufs obtenus jusqu'ici avec ces poules n'ont pas été assez satisfaisants pour faire des naines un concurrent sérieux prétendant à remplacer nos pondeuses commerciales actuelles.

Les recherches dans notre Département ont démontré (avec celles d'autres Instituts) que le niveau de production et la taille des œufs ne peuvent être influencés qu'à un degré très limité en changeant la composition de la ration des pondeuses. Dans notre laboratoire, des taux protidiques allant de I6 à $23 \mathrm{p}$. Ioo ont donné des résultats similaires sur des pondeuses naines. Si l'on considère le besoin énergétique théorique de l'animal nain (tenant compte de la taille corporelle plus petite et par suite des besoins d'énergie accrus par $\mathrm{kg}$ de poids), il apparaîtrait que l'énergie, plutôt que les protéines, serait le facteur limitant des naines. Cependant, l'augmentation du taux énergétique de la ration de 2,87 à 3,3 I kcal par g n'avait que peu ou pas d'effet sur la ponte ou la taille des œufs. L'accroissement de 25 p. Ioo (par rapport à la ration normale) des quantités de vitamines et des oligo-éléments et l'addition de DL-méthionine à la ration des pondeuses, de même, avait un effet faible ou nul sur les performances des poules mises en poulailler.

Afin de tester pourquoi les oiseaux nains ne répondent pas à des niveaux accrus des éléments de la ration, une recherche a été entreprise pour déterminer si la naine était un animal hypothyroïdien. Des poussins commerciaux normaux et des nains ont reçu des rations contenant divers taux de thyroprotéine et de thiouracil. La thyroprotéine avait pour effet une augmentation significative du poids corporel des naines pendant la période de croissance, ainsi qu'un allongement des tarses, aucun effet significatif n'étant noté avec les animaux normaux.

La température corporelle des oiseaux nains a été trouvée d'environ $0,5^{\circ} \mathrm{C}$, plus basse que celle des normaux. L'administration de thyroprotéine a eu pour résultat l'élévation de la température corporelle des nains au niveau de celle des normaux, tandis que le thiouracile produisait un abaissement de la température corporelle des normales et pas de changement chez les naines.

La composition des carcasses indiquait que les naines avaient une proportion de graisse corporelle significativement plus élevée que les normales. La thyroprotéine avait pour effet une diminution de la graisse des carcasses chez les naines, mais une augmentation chez les oiseaux normaux. L'addition de thiouracile aux rations conduisait à un taux de lipides plus élevé dans la carcasse des naines, mais à un taux diminué chez les normales. 
Il fut alors décidé d'assurer la croissance de poulettes naines avec une ration normale jusqu'à 20 semaines d'âge, puis d'essayer d'augmenter leur consommation alimentaire et par suite leur ponte et la taille de leurs œufs par l'adjonction de thyroprotéine dans l'aliment. Différents taux furent essayés avec peu ou pas de succès. Cependant, dans le dernier test, avec des taux aussi faibles que $0,009 \mathrm{p}$. Ioo, les performances des naines apparurent améliorées. D'autres travaux sont nécessaires pour tirer une conclusion définie dans ce domaine.

Une autre étude a été entreprise avec des rations à 16 et $19 \mathrm{p}$. I oo de protéines, contenant toutes deux des taux augmentés de vitamines et d'oligo-éléments ainsi qu'un supplément de méthionine. Il n'y a pas eu de différence en réponse aux traitements. Les pontes individuelles étaient enregistrées. La plupart des poules pondaient des séries de deux œufs ou moins. Ces résultats confirment des travaux rapportés antérieurement, suggérant que la taille des séries doit être améliorée avant que l'on puisse obtenir une amélioration majeure de la production.

Dans un autre travail, divers constituants sanguins des naines ont été comparés à ceux de Leghorn blanches normales. Il n'y avait pas de différence significative dans le volume des éléments cellulaires, l'hémoglobine, le nombre d'hématies, ou la concentration moyenne d'hémoglobine corpusculaire. Il en était de même pour le cholestérol total, les protéines ou l'albumine du sérum.

Les acides aminés du plasma ont été déterminés sur des poulets nains et normaux après 8 heures d'alimentation. La plupart ne présentaient pas de différence ; cependant, il y avait une décroissance marquée du taux de méthionine pour les nains comparés aux normaux (o, 78 contre 6 , ro $\mu \mathrm{mole} /$ roo $\mathrm{ml}$ de plasma). D'autres travaux sont en cours pour vérifier ce résultat.

\section{TABLE I}

Weight gain and feed consumption of dwarf birds to 8 weeks of age

Gain de poids et consommation alimentaire d'oiseaux nains jusqu'à 8 semaines d'âge

\begin{tabular}{|c|c|c|c|}
\hline \multicolumn{2}{|r|}{ Age } & \multirow{2}{*}{$\begin{array}{c}\text { Av. wt. } \\
(\mathrm{g})\end{array}$} & \multirow{2}{*}{$\begin{array}{c}\text { Av. weekly feed cons. } \\
\text { (g) }\end{array}$} \\
\hline Day old & $\ldots \ldots \ldots \ldots \ldots$ & & \\
\hline 1 week & $\ldots \ldots \ldots \ldots \ldots \ldots$ & 68 & 54 \\
\hline 2 weeks & $\ldots \ldots \ldots \ldots \ldots$ & 117 & 96 \\
\hline $3-$ & $\ldots \ldots \ldots \ldots \ldots \ldots$ & 159 & 109 \\
\hline- & $\ldots \ldots \ldots \ldots \ldots \ldots$ & 191 & 96 \\
\hline - & $\ldots \ldots \ldots \ldots \ldots \ldots$ & 233 & 117 \\
\hline - & $\ldots \ldots \ldots \ldots \ldots$ & 290 & 151 \\
\hline - & $\ldots \ldots \ldots \ldots \ldots \ldots$ & 356 & 177 \\
\hline 一 & $\ldots \ldots \ldots \ldots \ldots \ldots$ & 424 & 203 \\
\hline
\end{tabular}

TABLE 2

Performance of dwarf birds from 8 to 20 weeks of age

Performances des oiseaux nains de 8 à 20 semaines d'âge

\begin{tabular}{|c|c|c|}
\hline Treatment & $\begin{array}{l}\text { Av. body wt. } \\
\text { at } 20 \text { wks } \\
\text { (g) }\end{array}$ & $\begin{array}{l}\text { Av. feed cons. } \\
8-20 \text { weeks } \\
\text { (g) }\end{array}$ \\
\hline Cage L. E. $\ldots \ldots \ldots \ldots \ldots \ldots$ & 861 & 3406 \\
\hline Cage H. E. ........... & 863 & 3171 \\
\hline Floor L. E. . . . . . . . & 809 & 3169 \\
\hline Floor $H$. E. .......... & 824 & 3015 \\
\hline H. E. $\ldots \ldots \ldots \ldots \ldots \ldots$ & 844 & 3093 \\
\hline L. E. $\ldots \ldots \ldots \ldots \ldots \ldots$ & 835 & 3287 \\
\hline Floor $\ldots \ldots \ldots \ldots \ldots \ldots$ & 817 & 3093 \\
\hline Cage $\ldots \ldots \ldots \ldots \ldots \ldots$ & 862 & 3283 \\
\hline
\end{tabular}


TABLE 3

Experimental diets

Rations expérimentales

\begin{tabular}{|c|c|c|}
\hline Ingredients & 15 p. 100 & 23 p. 100 \\
\hline Yellow corn $\ldots \ldots \ldots \ldots \ldots \ldots \ldots$ & 66.5 & 42.0 \\
\hline Soybean meal $(50 \%) \ldots \ldots \ldots \ldots$ & 18.25 & 38.0 \\
\hline 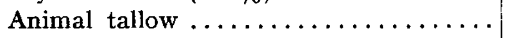 & 4.0 & 10.0 \\
\hline Ground limestone $\ldots \ldots \ldots \ldots \ldots \ldots$ & 6.75 & 7.25 \\
\hline Iodized salt $(0.015 \% \mathrm{KI}) \ldots \ldots \ldots \ldots$ & 0.25 & 0.25 \\
\hline Vitamin : mineral mix ............ & 1.0 & 1.0 \\
\hline DL-methionine $\ldots \ldots \ldots \ldots \ldots \ldots \ldots$ & - & 0.06 \\
\hline Alpha floc $\ldots \ldots \ldots \ldots \ldots \ldots \ldots$ & 1.25 & - \\
\hline \multicolumn{3}{|l|}{ Calculated analyses } \\
\hline Energy kcal ME/g .............. & 2.92 & 3.07 \\
\hline Calcium $(\%) \ldots \ldots \ldots \ldots \ldots \ldots$ & 3.03 & 3.20 \\
\hline Available phosphorus $(\%) \ldots \ldots \ldots$ & 0.55 & 0.50 \\
\hline
\end{tabular}

TABLE 4

Performance of dwarf hens from 23 to 53 weeks of age

Performances de poules naines de 23 à 53 semaines d'âge

\begin{tabular}{c|c|c|c}
\hline $\begin{array}{c}\text { Age } \\
\text { (weeks) }\end{array}$ & $\begin{array}{c}\text { Prod. H. D. B. } \\
(\%)\end{array}$ & $\begin{array}{c}\text { Av. feed/bird/day } \\
(\mathrm{g})\end{array}$ & $\begin{array}{c}\text { Av. egg weight } \\
(\mathrm{g})\end{array}$ \\
\hline & & & \\
23 & 3.0 & 43.5 & - \\
26 & 47.3 & 44.8 & 43.1 \\
33 & 60.2 & 61.2 & 50.3 \\
42 & 53.7 & 57.1 & 53.9 \\
50 & 50.8 & 54.8 & 57.0 \\
53 & 46.8 & 54.2 & 58.0 \\
\hline \hline
\end{tabular}

TABLE 5

Average weight gain and feed consumption

to 6 weeks of age for birds fed protamone and thiouracil

Gain de poids moyen et consommation d'aliment jusqu'à 6 semaines d'âge pour des poulets ayant ingéré de la protamone et du thiouracil

\begin{tabular}{|c|c|c|c|c|}
\hline \multirow{2}{*}{ Treatments } & \multicolumn{2}{|c|}{ Normal } & \multicolumn{2}{|c|}{ Dwarf } \\
\hline & $\begin{array}{l}\text { Av. wt. } \\
\text { (g) }\end{array}$ & $\begin{array}{c}\text { Feed/bird/day } \\
(\mathrm{g})\end{array}$ & $\begin{array}{l}\text { Av. wt. } \\
\text { (g) }\end{array}$ & $\begin{array}{c}\text { Feed/bird/day } \\
\text { (g) }\end{array}$ \\
\hline Control diet $\ldots \ldots \ldots \ldots \ldots \ldots \ldots$ & 376 & 20.6 & 267 & 14.4 \\
\hline Control $+0.033 \%$ protamone $\ldots \ldots \ldots$ & 388 & 23.4 & 335 & 19.6 \\
\hline Control $+0.05 \%$ thiouracil $\ldots \ldots \ldots \ldots$ & 314 & 17.8 & 210 & 11.5 \\
\hline Control $+0.075 \%$ thiouracil $\ldots \ldots \ldots$ & 293 & 15.7 & 188 & 10.5 \\
\hline
\end{tabular}


TABLE 6

Average shank length $(\mathrm{mm})$

for normal and dwarf birds to 6 weeks of age

Longueur moyenne des tarses $(\mathrm{mm})$

pour des oiseaux normaux et nains jusqu'à 6 semaines d'âge

\begin{tabular}{|c|c|c|c|c|}
\hline Treatments & Normal & $\begin{array}{c}\text { Deviation } \\
\text { from control } \\
(\%)\end{array}$ & Dwarf & $\begin{array}{c}\text { Deviation } \\
\text { from control } \\
(\%)\end{array}$ \\
\hline Control $\ldots \ldots \ldots \ldots \ldots \ldots \ldots \ldots \ldots$ & 7.30 & & 6.17 & \\
\hline Control $+0.033 \%$ protamone $\ldots \ldots \ldots$ & 7.56 & +3.6 & 6.80 & +10.2 \\
\hline Control $+0.05 \%$ thiouracil $\ldots \ldots \ldots$ & 6.73 & -7.8 & 5.86 & -5.0 \\
\hline Control $+0.075 \%$ thiouracil $\ldots \ldots \ldots$ & 6.00 & -17.8 & 5.42 & -12.2 \\
\hline
\end{tabular}

TABLE 7

Average body temperature $\left(\mathrm{C}^{\circ}\right)$ of normal and dwarf birds fed thyroprotein

Température corporelle moyenne $\left({ }^{\circ} \mathrm{C}\right)$ d'oiseaux normaux et nains recevant de la thyroprotéine

\begin{tabular}{|c|c|c|}
\hline & \multicolumn{2}{|c|}{8 weeks of age } \\
\hline & Normal & Dwarf \\
\hline \multirow[t]{3}{*}{$\begin{array}{l}\text { Control diet } \ldots \ldots \ldots \ldots \ldots \ldots \ldots \ldots \ldots \\
\text { Control }+ \text { protamone } \ldots \ldots \ldots \ldots \ldots \ldots\end{array}$} & $\begin{array}{l}41.6 \\
41.7\end{array}$ & $\begin{array}{l}41.0 \\
41.6\end{array}$ \\
\hline & \multicolumn{2}{|c|}{7 months of age } \\
\hline & Normal & Dwarf \\
\hline $\begin{array}{l}\text { Control } \operatorname{diet} \ldots \ldots \ldots \ldots \ldots \ldots \ldots \ldots \ldots \ldots \\
\text { Control }+ \text { protamone } \ldots \ldots \ldots \ldots \ldots \ldots\end{array}$ & $\begin{array}{l}41.5 \\
41.6\end{array}$ & $\begin{array}{l}41.1 \\
41.8\end{array}$ \\
\hline
\end{tabular}

\section{TABLE 8}

Carcass composition of 6 week old normal and dwarf birds (dry weight basis)

Composition de carcasse d'oiseaux normaux et nains âgés de 6 semaines (rapportée à la matière sèche)

\begin{tabular}{|c|c|c|c|c|}
\hline \multirow[b]{2}{*}{ Treatments } & \multicolumn{2}{|c|}{ Normal } & \multicolumn{2}{|c|}{ Dwarf } \\
\hline & $\begin{array}{c}\text { Protein } \\
(\%)\end{array}$ & $\begin{array}{l}\text { Fat } \\
(\%)\end{array}$ & $\begin{array}{c}\text { Protein } \\
(\%)\end{array}$ & $\begin{array}{l}\text { Fat } \\
(\%)\end{array}$ \\
\hline Control $\ldots \ldots \ldots \ldots \ldots \ldots \ldots$ & 53.8 & 29.7 & 51.1 & 35.7 \\
\hline Control $+0.033 \%$ protamone.. & 52.8 & 34.5 & 54.3 & 30.1 \\
\hline Control $+0.05 \%$ thiouracil $\ldots$ & 55.5 & 30.6 & 52.3 & 35.3 \\
\hline Control $+0.075 \%$ thiouracil .. & 56.6 & 25.6 & 47.9 & 38.5 \\
\hline
\end{tabular}


TABLE 9

Performance of dwarf and normal layers fed protamone

Performances de pondeuses naines et normales recevant de la protamone

\begin{tabular}{|c|c|c|c|c|c|c|}
\hline \multirow{2}{*}{ Treatments } & \multicolumn{2}{|c|}{ Prod. H. D. B. } & \multicolumn{2}{|c|}{$\begin{array}{c}\text { Av. feed/bird/day } \\
(\mathrm{g})\end{array}$} & \multicolumn{2}{|c|}{$\begin{array}{l}\text { Egg wt. } \\
\text { (g) }\end{array}$} \\
\hline & $\mathbf{N}$ & $\mathrm{D}$ & $\mathbf{N}$ & $\mathrm{D}$ & $\mathrm{N}$ & $\mathrm{D}$ \\
\hline Control $\ldots \ldots \ldots \ldots \ldots \ldots \ldots$ & 66.1 & 51.2 & 86 & 59 & 60 & 53 \\
\hline Control $+0.003 \%$ (P) $\ldots \ldots$ & 76.3 & 63.4 & 77 & 66 & 57 & 57 \\
\hline Control $+0.006 \%$ (P) $\ldots \ldots$ & 70.6 & 62.1 & 82 & 61 & 60 & 55 \\
\hline Control $+0.009 \%$ (P) $\ldots \ldots$ & 65.2 & 55.0 & 80 & 62 & 57 & 56 \\
\hline
\end{tabular}

(P) Protamone.

TABLE, IO

Number of hens laying at various rates of production Nombre de poules pondant à différents taux

\begin{tabular}{c|c|c|c|c}
\hline \hline \multirow{2}{*}{ Treatment } & $41-50 \%$ & $51-60 \%$ & $61-70 \%$ & $71-80 \%$ \\
\cline { 2 - 4 } & & & & \\
\hline 1 & 1 & 5 & 3 & 6 \\
3 & 1 & 2 & 5 & 5 \\
4 & 1 & 4 & 9 & 2 \\
& & 3 & 6 & 4 \\
\hline
\end{tabular}

TABLE II

Influence of diet on size of egg clutch

Influence du régime sur la taille des séries (clutchs) de ponte

\begin{tabular}{|c|c|c|c|c|c|}
\hline \multirow{2}{*}{ Treatment } & \multicolumn{5}{|c|}{ Size of clutch } \\
\hline & 2 or less & 3 & 4 & 5 & 6 \\
\hline $1 *$ & 328 & 109 & 42 & 9 & 5 \\
\hline 2 & 321 & 92 & 47 & 22 & 8 \\
\hline 3 & 381 & 96 & 36 & 10 & 3 \\
\hline 4 & 380 & 114 & 28 & 5 & 4 \\
\hline
\end{tabular}

* 16 birds $\times 112$ days. 


\section{TABLE I2}

Mean values for ved blood cell parameters of dwarf and non-dwarf 16 month old hens

Valeur moyenne des paramètres sanguins de poules naines et non-naines âgées de 16 mois

\begin{tabular}{l|c|c|c|c}
\hline \hline & $\begin{array}{c}\text { Packed cell } \\
\text { volume }\end{array}$ & Hemoglobin & $\begin{array}{c}\text { Red blood } \\
\text { cells } \\
(\%)\end{array}$ & $\begin{array}{c}\text { Mean corpuscular } \\
\text { hemoglobin } \\
\text { concentration } \\
(\%)\end{array}$ \\
\hline & $(\%)$ & $(\mathrm{ml} / \mathrm{cm})$ & \\
\hline Dwarf ..... & 27.7 & 8.5 & 2.93 & 30.7 \\
Non-dwarf... & 23.4 & 9.2 & 2.87 & 39.3 \\
\hline \hline
\end{tabular}

TABLE I3

Blood serum constituents of dwarf and non-dwarf 16 month old hens

Constituants du sérum sanguin de poules naines et non-naines âgées de 16 mois

\begin{tabular}{l|c|c|c|c}
\hline & $\begin{array}{c}\text { Total } \\
\text { cholesterol } \\
(\%)\end{array}$ & $\begin{array}{c}\text { Total } \\
\text { serum } \\
\text { protein } \\
(\%)\end{array}$ & $\begin{array}{c}\text { Serum } \\
\text { albumin } \\
(\%)\end{array}$ & $\begin{array}{c}\text { Albumin : } \\
\text { globulin } \\
\text { ratio }\end{array}$ \\
\hline & $\ldots$ & 4.0 & 49.2 & 0.97 \\
Dwarf ...... & 123.3 & 4.2 & 40.6 & 0.68 \\
\hline
\end{tabular}

